\title{
Urban Space with Instant and Ubiquitous Access Technologies ${ }^{\mathrm{i}}$
}

\author{
Critical Question: How Could Emerging Ubiquitous Access Technologies Help us to Achieve a \\ Sustainable Global Urban System in a Ubiquitous Technology Space?
}

\author{
Tschangho John Kim \\ University of Illinois at Urbana-Champaign, Champaign, USA \\ E-mail: tjohnkim@illinois.edu \\ Received April 9, 2011; revised May 7, 2011; accepted May 16, 2011
}

\begin{abstract}
According to the United Nations, the world will need to build new cities and/or expand existing cities to accommodate about 1.6 billion additional urban residents by 2030. This rapid trend is the result of many complex socio-economic and political factors, and poses unprecedented challenges to the functioning of cities and the quality of life for urban dwellers. The resources needed for accommodating new urban dwellers will be enormous. Can emerging information, communication and ubiquitous access technologies help us to achieve a sustainable global urban system in a ubiquitous technology space, mitigating the consumption of scarce resources?
\end{abstract}

Keywords: Ubiquitous Access Technology, Ubiquitous Technology Space, Sustainable Global Cities, Future Infrastructure

\section{Introduction}

The ability of individuals to interact and participate in activities relies on location information and access to it. As interactions among individuals become more complex as in urban areas, there has been and will be ever growing need for location and access information. These information will play vital roles in providing effective collaboration for personal and social productivity, and ultimately affect the socio-economic and behavioral patterns in an urban area.

A plethora of information and communication technologies (ICTs) are available, and alternative new technologies are emerging. Ubiquitous access technologies are relatively new sets of concepts, practices, and standards. The emergence of the technologies need to be understood within a broader context of both paradigm changes in computing technologies-from centralized to distributed, mobile, and ubiquitous computing - and the advancement of ICTs including ubiquitous geographic information (UBGI), radio-frequency identification (RFID), location-based services (LBS), global positioning systems (GPS) and sensing technologies.

The advancement of ICTs in last decades brought a negative impact on society, the digital divide. Before the division, most people would share much of the same information. But now people without access to ICTs can be isolated living in non-intersecting worlds with distinct sets of socio-economic patterns. Ubiquitous access technologies will positively impact society on a large scale if it becomes pervasive, by allowing more people in more places to have access to more powerful tools. These technologies could motivate people to interact with one another and digital apartheid typically segregating space and places could be integrated.

The problems caused by greater dispersal of urban activities, which have led to increased distances from home to jobs, shops, schools and leisure facilities, can be mitigated by pervasive use of ubiquitous access technologies. Congestion caused urban Americans to travel 4.2 billion hours more and to purchase an extra 2.9 billion gallons of fuel for a congestion cost of $\$ 78$ billion in 2005. In addition to this cost in time and money, traffic that is slowed by congestion causes a great deal of pollution. These emissions kill 30,000 people each year ${ }^{1}$ in the U.S., and car collisions kill an additional 40,000 in 2007.

${ }^{1}$ http://bicycleuniverse.info/transpo/almanac.html [Accessed on October 5, 2010] 


\section{Foreseeable Scenarios}

Pervasive use of these technologies could save resources in the forthcoming decades of global urbanization. By 2010, more than half the world's population live in urban areas and the world population will reach 8.2 billion by 2030 with more than 60 percent of this total expected to live in urban areas. This means it is necessary to build new cities over the next 25 years, or to expand the existing metropolitan areas to become megacities to accommodate 1.65 billion additional urban residents. This rapid trend are the result of many complex economic, social, demographic, and political factors and pose unprecedented challenges to the functioning of cities and the quality of life for urban dwellers. The resources needed for accommodating new urban dwellers will be enormous. An important question is can an urban area be sustainable?

The full deployment of ubiquitous access technologies, coupled with other ICTs, would reduce energy consumption, and would decrease particulate emissions and congestion in cities by finding and providing an optimized path between origins and destinations, through delivering and receiving such path information services wirelessly, thus mitigating unnecessary travel. These behavioral changes can also reduce the side effects of travel such as greenhouse gases, accidents, and superfluous transportation infrastructure. The advancement of mobile and stable sensing and access technologies, along with new widespread economic activities generated and governed by information networks, provide scientists and engineers with powerful tools for preparing and supporting innovative urban strategies that promote sustainability and informed decision-making. These promise increased convenience, awareness, transparency, and access to information, as well as social opportunities that break with traditional power structures yet reduce the consumption of scarce resources by receiving and delivering services anywhere at any time in a ubiquitous technology space.

\section{Challenges}

Scientists and engineers, and particularly urban planners and geographers, have been searching for ways to dramatically transform the way the urban environment is designed, managed, and run. As policy for urban management moves from a centralized, top-down approach to a decentralized and bottom-up perspective, our conception of urban systems needs to be changed [1]. The instant and ubiquitous access technologies could greatly increase the ability of scientists to create transformative changes in the consumption of scarce urban resources and energy use to reduce pollution with minimum social costs. Defining urban sustainability as acceptable levels of social costs associated with the daily activities and physical movements of people and goods, then the key social costs that burden the economy at large are related to the use and misuse of scarce urban land, the decay of environmental quality, traffic accidents, and traffic congestion. Instant and ubiquitous access technologies could be key in helping us to achieve a sustainable global urban system in a ubiquitous technology space.

Advances in science and technology, particularly in the past century, have significantly contributed to the evolution of cities and welfare of citizens [2]. In recent decades, many important new discoveries spearheaded by evolution of ICTs have contributed enhancing the quality of life for millions of individuals. However, the potential for science and technology to ameliorate or solve the problems of the world's multiplying cities has not been realized. A much broader and interdisciplinary research is needed on how the range of existing technological and scientific research findings can be translated into actions at the national, regional and local levels.

The main research agenda for future urban settlements is to conserve scarce resources by utilizing the ubiquitous access and the emergence of pervasive ICTs and to identify ways for existing cities to grow in a more sustainable and intelligent manner. The rapid convergence of ubiquitous and cloud computing technologies, and ICTs is raising the possibility of a dramatic transformation in the way we perceive and move about the urban environment, and how we interact with each other in urban spaces. Facing the following paradigm shifts from the traditional urban space to the ubiquitous technology space would be challenging tasks for scientists and engineers:

- From street-oriented to information-oriented,

- From economy of scale to economy of scope,

- From centralized information to distributed information,

- From standardization to customization,

- From efficiency-oriented to quality-oriented,

- From regularity to flexibility, and

- From distance-oriented to time-oriented.

\section{Unanswered Questions}

Toward answering the underlying critical question of how could emerging ubiquitous access technologies help us to achieve a sustainable global urban system in a ubiquitous technology space, a host of challenging questions should be answered. Examples of such questions are:

1 Would the instant and ubiquitous access technologies and other ICTs become centripetal or centrifugal forces that affect the shape of urban areas? 
2 Will the ubiquitous technologies change research domain for human settlements, particularly in urban areas, and if so how?

3 Will the scarce urban resources be saved, and if so, how much?

4 What will be changes in individual behaviors and what will be implication of such changes on collective behavior in urban areas?

5 What would be implications to the public policy?

6 Will the ubiquitous access technologies enhance the function of city or eliminate face-to-face interactions and make cities obsolete?

7 Will ubiquitous access technologies help with reduction of the use fossil oil and enhance the environment, and if so, how much?

\section{Capacity Building}

The improvement of existing cities, as well as planning for future urban settlements, needs to become a new priority discipline in which expertise is developed by interdisciplinary collaborations among a wide range of disciplines, including the physical sciences, urban planning, engineering, human health, ecology, economics, geography, architecture, sociology, political science and computer science. This part of the white paper is heavily drawn from the statement by seventy-two of the World's Scientific Academies ${ }^{2}$.

Cross-disciplinary Education and Training: The nature and complexity of challenges of urban settlements inherently require integrated efforts among education, research, and operational institutions. A new set of crossdisciplinary curricula will educate a cadre of future social scientists, engineers and urban planners. Inter-disciplinary research is necessary not only to produce new

\footnotetext{
${ }^{2}$ Science and Technology and the Future of Cities, A Statement by Seventy-Two of the World's Scientific Academies, June 1996, from http://www.interacademies.net/?id=3551 [Accessed on October 5, 2010].
}

knowledge, but to build the capacity to assess, absorb, and use technology and experience developed elsewhere.

Non-technological Institutional Elements: A new set of challenging non-technical issues includes legal frame works, including property ownership and the protection of intellectual property, effective and flexible standards, institutions for efficient mobilization of capital resources, tax and regulatory structures which are conducive to innovative solutions to urban challenges.

International Cooperation: The sustainability of cities in the decades to come requires a better understanding of the complex interactions among environmental, economic, political, social and cultural factors at local, regional, and global levels. Global urban problems cannot be solved in isolation. Developing and sharing information and technologies will be necessary since innovative solutions developed in one city can be applied to other cities. International assistance should be provided for capacity building of local scientific and technological expertise. The worldwide scientific community should work together and with political and managerial decision makers to identify and implement innovative solutions for meeting the challenges faced by the rapid global urbanization trend and the emerging ubiquitous access and other related technologies.

\section{References}

[1] Michael Batty, "Cities and Complexity," The MIT Press, Cambridge, Massachusetts, 2005.

[2] Tschangho John Kim, Matthew Claus, Joseph S. Rank and Yu Xiao, "Technology and Cities: Processes of Technology-Land Substitution in the 20th Century," Journal of Urban Technology, Vol. 16, No. 1, 2009, pp. 63-88.

[3] Harvey J. Miller, "Societies and Cities in the Age of Instant Access,” Springer, Berlin, 2007.
${ }^{\mathrm{i}}$ This is a revised version of a white paper submitted to the National Science Foundation as a possible future research for 2020 in the Social, Behavioral and Economic Sciences. All other white papers submitted to NSF in 2010 can be seen in http://www.nsf.gov/sbe/sbe 2020/all.cfm. 